\title{
Isolation of Respiratory Syncytial Virus from Middle Ear Exudates of Infants
}

\author{
BO BERGLUND, ALTTI SALMIVALLI, PAAVO TOIVANEN, and JOHAN WICKSTRÖM \\ From the Department of Virology, the Otolaryngological Clinic, the Department of Medical Microbiology, and the \\ Childrens' Clinic, University of Turku, and from the Hospital for Infectious Diseases, Turku, Finland
}

During an epidemic of respiratory infection due to respiratory syncytial (RS) virus in Turku, Finland, which lasted from the beginning of April to the end of June 1965, the virus was isolated for the first time from the middle ear exudates of two children with otitis media (Berglund and Stråhlmann, 1966). This report describes further isolations of RS virus from the ears of several infants with middle ear infection, using improved techniques.

A more detailed report of this study has been published elsewhere (Berglund, Salmivalli, and Toivanen, 1966).

\section{Materials and Methods}

A total of 27 children, ranging in age from 2 months to 4 years with mild to severe symptoms or signs of middle ear infection were studied. All were either patients in the wards of the Children's Clinic, or attending the Outpatient Clinic of Otolaryngology. The study was conducted over a 16-day period in June 1965.

Specimens from the middle ears were obtained with a syringe and a needle by a puncture aspiration technique described by Lahikainen (1953). No anaesthesia was used for those under the age of 12 months; older children were given general divinyl ether anaesthesia. Cleaning procedures were confined to mechanical cleansing of the external meatus with a stick dipped in alcohol.

After puncture of the tympanic membrane one drop of the exudate was immediately injected into a tube containing a monolayer culture of a continuous cell line of human amnion ( $U$ cells) grown in a $0.5 \%$ lactalbumin hydrolysate medium in Hanks's solution supplemented with $25 \%$ calf serum and maintained in $2 \mathrm{ml}$. Eagle's minimum essential medium, containing 5\% inactivated horse serum, $5 \%$ tryptose phosphate, 800 units of penicillin, $800 \mu \mathrm{g}$. streptomycin, 25 units nystatin, and $5 \mu \mathrm{g}$. amphotericin B per ml. Bacteriological examinations were carried out on the middle ear exudates remaining in the syringes according to a method described elsewhere (Grönroos, Kortekangas, Ojala, and Vuori, 1964). After taking samples from the middle ear

Received March 4, 1966. exudates, duplicate $U$ cell cultures were inoculated each with one of two throat swab specimens by a direct inoculation method (Berglund, Vihma, and Wickström, 1965). The cell culture tubes were maintained at $35^{\circ} \mathrm{C}$. for 2 weeks and the medium was changed twice a week.

Paired serum specimens were obtained within an interval of 2 to 5 weeks from the children in hospital. For titration of the sera the micro-complement-fixation (CF) method of Sever (1962) was used with minor modifications. Three antigens were employed in the $\mathrm{CF}$ tests: one from the Randall strain, one from a virus isolate obtained from the ear producing syncytial degeneration, and a cell control antigen. All antigens were prepared in $U$ cells, grown and maintained as described above. The antigens were titrated against 8 antibody units of a serum pool consisting of early convalescent phase RS virus-immune sera from 4 children. 4 units of antigen and 2 full units of complement were employedo in the titration of the sera. A control complement: titration and a serum control, as well as a titration of a serum with known Randall strain RS virus CF antibody titre were included in each test. Neutralization tests for the identification of RS virus were carried out in $U$ cells using hyperimmune guinea-pig serum, prepared against the Randall strain of RS virus.

\section{Results}

Children whose throat swab specimens contained RS virus. RS virus was isolated from the throat swab specimens of 10 out of 27 children. Except for one child, aged 1 year 9 months, who visited the out-patient clinic of otolaryngology, all 10 children were under the age of 12 months and were in hospital for a lower respiratory tract illness. Their only sign of middle ear affection appeared to be stiffness of the tympanic membrane. The exudate obtained by aspiration puncture was serous or mucous and clear. Puncture of the middle ear was carried out bilaterally in 7 infants and unilaterally in 3. Of those in whom puncture was performed bilaterally, 4 children were found to have RS virus in both ears, 2 in one ear, and one (aged 1 year and 9 months, and mentioned above) had no virus in her ears. RS virus was found in the 
middle ear exudates of all those 3 in whom the puncture was performed unilaterally.

Repeated aspiration punctures were carried out on 5 of these infants 2-7 days after the first puncture, and at the time of the second puncture virus could no longer be found in the middle ear exudates, though in 3 of the 5 babies the throat swab specimens still contained virus.

Bacterial culture of the middle ear exudates was either negative or yielded Staphylococcus albus. This bacterium, usually considered non-pathogenic for the middle ear, was probably derived from the external acoustic meatus. All but one of the children received treatment with antibiotics. Only one exudate (obtained at the second puncture) was found to contain a potentially pathogenic bacterium, Staphylococcus aureus.

All virus isolations identified as RS viruses produced typical syncytial degeneration in the cell cultures. They were completely neutralized by the Randall strain RS virus immune serum, provided the amount of virus used in the neutralization tests did not exceed $100 \mathrm{TCD}_{50}$ units.

Appropriate paired serum specimens were available for 7 children. 3 responded with a fourfold or greater rise in the RS virus CF antibody titre, and 2 responded with a rise in titre from $<1 / 4$ to $1 / 4$. One showed a twofold fall in titre from $1 / 32$ to $1 / 16$, and one an equally high titre of $1 / 32$ in the two serum specimens. Titres of the same level were obtained by both RS viral antigens.

Children whose throat swab specimens did not contain RS virus. The throat swab specimens of 17 children, aged 2 months to 4 years, were negative for RS virus. Aspiration puncture of the ears was performed bilaterally in 11 children and unilaterally in 6 . No RS virus was isolated from the middle ear exudates, whereas potentially pathogenic bacteria (Haemophilus influenzae, Diplococcus pneumoniae, Neisseria catarrhalis, Strepto- coccus pyogenes, and Strep. viridans) were found on culture in the exudates of 11 children (65\%). The exudates of 6 children were negative, or contained only Staphylococcus albus. Most of the 17 children were out-patients of the clinic and half of them were over 1 year old. The symptoms and signs of middle ear infection were on the whole more severe. 6 were treated with antibiotics at the time of the puncture.

\section{Summary and Conclusions}

Specimens of exudate from the middle ear were obtained by puncture of the drum from 27 children during a respiratory syncytial (RS) virus epidemic in Turku, Finland, in the spring and early summer of 1965 . By direct virus inoculation RS virus was recovered from one or both ears of 9 out of 10 infants suffering from a lower respiratory tract illness caused by $R S$ virus.

It is probable that RS virus causes a mild, often bilateral middle ear infection in infants, with accumulation of exudate and decreased mobility of the drum. However, it is also possible that exudate containing virus is merely conducted mechanically from the nasopharynx to the middle ear without producing an actual virus infection of the middle ear epithelium.

\section{REFERENCES}

Berglund, B., Salmivalli, A., and Toivanen, P. (1966). Isolation of respiratory syncytial virus from middle ear exudates of infants. Acta oto-laryng. (Stockh.), 61, 475.

-, and Stråhlmann, C-H. (1966). In preparation.

- Vihma, L., and Wickström, J. (1965). Respiratory syncytial virus studies on children hospitalized during an outbreak of respiratory illness in Finland. Amer. F. Epidem., 81, 271.

Grönroos, J. A., Kortekangas, A. E., Ojala, L., and Vuori, M. (1964). The aetiology of acute middle ear infection. Acta oto-laryng. (Stockh.), 58, 149.

Lahikainen, E. A. (1953). Clinico-bacteriologic studies on acute otitis media. Aspiration of the tympanum as a diagnostic and therapeutic method. ibid., Suppl. 107.

Sever, J. L. (1962). Application of a microtechnique to viral serological investigations. $\mathcal{F}$. Immunol., 88, 320. 